\title{
Expectativas de estudiantes universitarios hacia el aprendizaje de metodología científica
}

\section{Expectations of University students towards learning of scientific methodology}

\section{Expectativas de estudantes universitários para a aprendizagem de metodologia científica}

\author{
Pedro César Cantú-Martínez*, Gabriela Robles Camporredondo*, \\ José Antonio Heredia Rojas ${ }^{* * *}$, Abraham O. Rodríguez de la Fuente ${ }^{* * * *}$, \\ Libertad Leal Lozano ${ }^{* * * *}$, Laura Ernestina Rodríguez Flores ${ }^{* * * * *}$, María Es- \\ peranza Castañeda Garza ${ }^{* * * * * *}$, Martha Alicia Santoyo Stephano ${ }^{* * * * * * *}$
}

Fecha de recepción: 19 de junio de 2016

Fecha de evaluación: 27 de junio de 2016

Fecha de aceptación: 8 de mayo de 2017

Disponible en línea: 18 de mayo de 2017

DOI: http://dx.doi.org/10.18359/reds.1937

Cómo citar este artículo:

Cantú-Martínez, P.C., Robles-Camporredondo, G., Heredia-Rojas, J.A., Rodríguez-de la Fuente, A.O., Leal-Lozano, L., Rodríguez-Florez, L.E., Castañed-Garza, M.E. y Santoyo Stephano, M.A. (2017). Expectativas de estudiantes universitarios hacia el aprendizaje de metodología científica. Revista Educación y Desarollo Social, 11(1), 74-84. DOI: org/10.18359/reds.1937.

Doctorado en Ciencias Biológicas, de la Universidad Autónoma de Nuevo León, Facultad de Ciencias Biológicas, Miembro del Cuerpo Académico 181 Ciencias Exactas y Desarrollo Humano. San Nicolás de los Garza, N.L., México. Correo electrónico: pedro.cantum@uanl.mx, cantup@hotmail.com

** Licenciada en Biotecnología Genómica, de la Universidad Autónoma de Nuevo León, Facultad de Ciencias Biológicas, San Nicolás de los Garza, N.L., México. Correo electrónico: gaby-roblesc14@hotmail.com

*** Doctorado en Biología Celular y Genética, de la Universidad Autónoma de Nuevo León, Facultad de Ciencias Biológicas, Miembro del Cuerpo Académico 181 Ciencias Exactas y Desarrollo Humano. San Nicolás de los Garza, N.L., México. Correo electrónico: jherediarojas@gmail.com

**** Doctorado en Ciencias en Microbiología, de la Universidad Autónoma de Nuevo León, Facultad de Ciencias Biológicas, Miembro del Cuerpo Académico 181 Ciencias Exactas y Desarrollo Humano. San Nicolás de los Garza, N.L., México. Correo electrónico: aor@@hotmail.com

***** Doctorado en Ciencias, de la Universidad Autónoma de Nuevo León, Facultad de Ciencias Biológicas, San Nicolás de los Garza, N.L., México. Correo electrónico: libertad.leallz@uanl.edu.mx

****** Maestría en Ciencias, de la Universidad Autónoma de Nuevo León, Miembro del Cuerpo Académico 181 Ciencias Exactas y Desarrollo Humano. Facultad de Medicina, Monterrey, N.L., México. Correo electrónico: lardzflor@hotmail.com

$74 \quad$ Miembro del Cuerpo Académico 181 Ciencias Exactas y Desarrollo Humano. San Nicolás de los Garza, N.L., México. Correo electrónico: perycas@yahoo.com

******** Maestría en Enseñanza Superior, de la Universidad Autónoma de Nuevo León, Miembro del Cuerpo Académico 181 Ciencias Exactas y Desarrollo Humano. Facultad de Ciencias Biológicas, San Nicolás de los Garza, N.L., México. Correo electrónico: marthastephano@yahoo.com.mx 


\section{Resumen}

Este artículo examina las expectativas de estudiantes universitarios de una Facultad de Ciencias Biológicas hacia la unidad de aprendizaje metodología científica, con el fin de verificar sus expectativas antes y después del curso. Es un estudio transversal y exploratorio de 2012 a 2014, que se llevó a cabo mediante una encuesta con 10 ítems que se aplicó a 237 alumnos en primera instancia, y posteriormente a 177 que cursaron la asignatura de Metodología Científica. Se verificó que el programa estimula en los estudiantes los niveles cognoscitivos de comprensión, conocimiento, análisis y aplicación; asimismo, que el género y la carrera que cursaban los participantes no determinan diferencias significativas. Se encontró, además, en relación con la historia y filosofía de la ciencia que no se cumplen las expectativas para la ejercitación de la elaboración de un protocolo de investigación y búsqueda de información. Los participantes aseveran de forma general que esto debe impartirse a todos los estudiantes de una Facultad de Ciencias. Es importante considerar que la ciencia y sus métodos están en una constante estructuración del conocimiento y están vinculados con la realidad en espacio y tiempo; por lo tanto, es pertinente conocer las percepciones de los estudiantes sobre una asignatura de aprendizaje.

Palabras clave: expectativas, estudiantes universitarios, metodología científica, ciencia.

\section{Abstract}

This article examines the expectations of university students from a Faculty of Biological Sciences towards the unit of learning scientific methodology, to verify their expectations before and after the course. It is a cross-sectional and exploratory study from 2012 to 2014, which was carried out through a 10-item survey that was applied to 237 students in the first instance, and then to 177 who completed the Scientific Methodology course. It was verified, that the program stimulates in students cognitive levels of understanding, knowledge, analysis and application and that the gender and career of the participants did not determine significant differences. It was also found about the history and philosophy of science that the expectations for the exercise of the elaboration of a protocol for investigation and search of are not met. Participants assert that this should be imparted to all students of a Faculty of Sciences. It is important to consider that science and its methods are in a constant structuring of knowledge and are linked to reality in time and space; therefore, it is pertinent to know the perceptions of students on a course of learning.

Keywords: expectations, university students, scientific methodology, science.

\section{Resumo}

Este artigo analisa as expectativas dos estudantes universitários de uma Faculdade de Ciências Biológicas para a unidade de aprendizagem metodologia científica, a fim de verificar as suas expectativas antes e após o curso. É um estudo transversal e exploratório de 2012 a 2014, realizado através de uma sondagem com 10 itens e foi aplicado a 237 alunos, em primeira instância, e depois para 177 que completaram o curso de metodologia científica. Verificou-se que o programa estimula nos estudantes os níveis cognitivos de compreensão, conhecimento, análise e aplicação; também que o gênero e a carreira que cursavam os participantes não determinam diferenças significativas. Encontrou-se, além disso, em relação com a história e filosofia da ciência que não são cumpridas as expectativas para o exercício da elaboração de um protocolo de pesquisa e procura de informação. Os participantes afirmam de maneira geral que isto se deve ministrar a todos os alunos de uma Faculdade de Ciências. É importante considerar que a ciência e seus métodos estão em uma constante estruturação do conhecimento e estão ligados com a realidade em espaço e tempo; portanto, é pertinente conhecer as percepções dos alunos sobre um curso de aprendizagem.

Palavras-chave: expectativas, estudantes universitários, metodologia científica, ciência. 


\section{Introducción}

En los inicios del nuevo siglo, se ha contemplado el relevante papel que la educación superior tiene en la sociedad mexicana, particularmente en la actuación que esta asume para el desarrollo cultural, social y económico. Esto esencialmente para preparar nuevas sucesiones de ciudadanos instruidos con competencias y nuevos conocimientos, para hacer frente a los desafíos y oportunidades que significan los distintos avances tecnocientíficos en los ámbitos de generación, estructuración, codificación, transmisión y acercamiento al conocimiento científico (Gutiérrez Vargas, 2002; Tamez-Guerra y Martínez-Rizo, 2012). Así es como la representación que trasmite la educación superior es la de constituirse en un espacio que subsiste para la generación de conocimiento y reproducción de trabajo, semejante o más superior al que rige en el ámbito de la producción y de la sociedad misma (Rué, 2009).

En este sentido, la investigación se erige como la principal instrumentación que permite el impulso y desarrollo de la educación superior, lo cual puede observarse mediante el ejercicio que realizan los académicos al efectuar investigación básica como aplicada. La primera promueve y fortalece el conocimiento de frontera, mientras que la segunda utiliza el conocimiento para sustentar intervenciones y resolver situaciones que aquejan a la sociedad. Recordemos, como señala Cantú-Martínez (2012),
"La ciencia se constituye como la principal forma de generar conocimiento, y desempeña una función trascendente en nuestra sociedad, a través de su primordial actividad, que es la investigación científica" (p. 12).

Sin embargo, además de la investigación realizada por los profesores universitarios la cual es de suma importancia, las instituciones de educación superior han tenido que incorporar en sus planes de estudios procesos de enseñanza-aprendizaje para que los estudiantes lleven a cabo ejercicios académicos que los acerquen a los procesos formales de la investigación científica durante su formación profesional (Díaz, 2011). Esto con la finalidad de ampliar en los alumnos su panorama intelectual y potencialmente abrir oportunidades laborales, cuando se pasa del nivel formativo al productivo.

Con el reconocimiento de lo anterior, la Universidad Autónoma de Nuevo León (2005) crea el Área Curricular de Formación General Universitaria, para ofrecer un espacio donde los estudiantes desarrollen competencias acordes con las necesidades sociales y de orden laboral. Entre las materias que integran esta área curricular se encuentra la asignatura de Metodología Científica, cuyo propósito es desarrollar en el estudiante la capacidad de valorar y apreciar la aportación de la ciencia al bienestar del ser humano. De acuerdo con Benomo Alciaturi et al. (2006), esto "contribuye al estudiante, futuro profesional, a par- 
ticipar activamente en el campo de la investigación" (p. 1), y como lo señala Briceño (1999, citado por Scandroli y Eyler, 2007) "[...] ahora se debe pasar al diseño y concreción de experiencias que coloquen a los estudiantes en situaciones similares a las que han vivido las comunidades científicas a lo largo de determinados procesos de elaboración del saber" (p. 2).

De esta manera, se espera que el "estudiante no interesado en la investigación científica tenga al menos un nivel básico en relación con todos sus procesos" (Molina Ordoñez et al., 2008, p. 325), con la finalidad de que los alumnos adquieran una conveniente comprensión del quehacer científico.

Petrucci y Dibar Ure (2001) indican que el tema relacionado con las expectativas que los estudiantes exteriorizan hacia los cursos de enseñanza de las ciencias es una de las vertientes que abarcan los estudios relativos a la naturaleza de la ciencia. Con este fin se lleva a cabo la presente indagación de carácter exploratorio, con la finalidad de conocer los propósitos y contenido de la asignatura, así como las expectativas de los estudiantes universitarios del área de ciencias biológicas hacia la asignatura de Aprendizaje de Metodología Científica.

\section{Metodología}

La presente investigación de carácter transversal y exploratorio se llevó a cabo entre 2012 y 2014, con estudiantes de la Facultad de Ciencias Biológicas, de la Universidad Autónoma de Nuevo León (Nuevo León, México). La muestra fue aleatoria y correspondió a 237 alumnos de tercer semestre de ambos géneros, que pertenecen a las cuatro carreras que se imparten en la dependencia, como son Biólogo (BIOL), Químico Bacteriólogo Parasitólogo (QBP), Licenciado en Ciencias de los Alimentos (LCA) y Licenciado en Biotecnología Genómica (LBG). Estos se encontraban cursando de forma regular su asignatura de Metodología Científica. Se recabó información relacionada con su género, edad y carrera cursada; además, se llevó a cabo una encuesta ad hoc con 10 items en los que se exploró acerca de lo que esperaban los estudiantes aprender y cómo esperaban ejercitarse en la unidad académica, así como quiénes consideraban que debería cursar esta, en dos momentos: al inicio y final del curso. Se exploró también su involucramiento en aspectos de investigación científica previamente. Se realizó el examen del plan de estudio de la asignatura y se contrastó con la taxonomía de Bloom (Campos Arenas, 2007; Woolfolk, 2010). Con la información recabada se integró una base de datos para ser analizados en el programa estadístico SPSS.

\section{Resultados}

Propósitos y contenido de la asignatura

El programa de Metodología Científica señala en sus propósitos cuatro 
declaraciones que desea que el alumno manifieste: 1) distinguir entre el conocimiento empírico y aquel que se obtiene metódicamente; 2) identificar los principales aspectos en un diseño de investigación; 3) analizar la organización básica de un proyecto de investigación científica, y 4) realizar una propuesta de protocolo de investigación científica. Como se observa en las enunciaciones citadas, se pueden caracterizar los fines educacionales del programa educativo, tomando en cuenta los verbos que declaran "distinguir-identificar-analizar-realizar" y relacionar estos con los fines cognoscitivos de enseñanza advertidos en la taxonomía de Bloom permitió transcribirlos en los niveles cognoscitivos de "comprensión-conocimiento-análisis-aplicación", respectivamente; de esta manera, se hallaron cuatro de los seis dominios que establece esta taxonomía.

En relación con el contenido de la asignatura, esta contiene aspectos temáticos por tratar, como son: 1) desarrollo histórico de la ciencia; 2) ciencia y método científico; 3) etapas del proceso de investigación; 4) protocolo de investigación; 5) diseño metodológico y estadístico; 6) plan de trabajo; 7) elaboración de un protocolo de investigación, y 8) presentación de reportes de investigación. Aquí se pudo percatar que no más del $25 \%$ de los propósitos de aprendizaje en la asignatura se refieren a conocimiento, es decir, solo a recordar información aprendida, advirtiendo que el $75 \%$ del programa restante refiere a la interpre- tación de información, segmentación del conocimiento y examinación de las relaciones en el conocimiento para construirlo de nueva cuenta en nuevas situaciones, en términos de operaciones de pensamiento y conductas observables.

Expectativas de los estudiantes al inicio del curso

De los 237 estudiantes participantes encuestados, el 38,4\% ( $\mathrm{f}=91$ ) corresponde a la carrera de QBP; le siguen las carreras de LBG, BIOL y LCA, con $36,7 \%(\mathrm{f}=87), 21,9 \%(\mathrm{f}=52)$ y $3 \%(\mathrm{f}=7)$, respectivamente. En cuanto al género, un $53,6 \%(\mathrm{f}=127)$ fueron mujeres y un $46,4 \%$ ( $\mathrm{f}=110)$ fueron varones, mientras la edad de los participantes fluctuó entre los 17 años y 31 años, con un promedio de 18,7 años, con una variación de los datos con respecto al valor medio de 1,6 años. Con referencia a las preguntas consideradas en la encuesta, cuando se les cuestionó si esperaban aprender historia de la ciencia, el 71,3\% ( $\mathrm{f}=169)$ indicó que sí, mientras que $28,7 \%$ ( $\mathrm{f}=68$ ) mencionó que no. Un comportamiento muy similar se pudo observar al cuestionarles si esperaban aprender aspectos relacionados con la filosofía de la ciencia, donde $66,7 \%(\mathrm{f}=158)$ y $33,3 \%(\mathrm{f}=79)$ respondieron correspondientemente que sí y no. Cuando se les argumentó sobre si esperaban ejercitarse en la elaboración de un protocolo de investigación, el 99,6\% (f=236) señaló que sí, mientras que al preguntarles si esperaban adiestrarse en la búsqueda de material bibliográfico para el apoyo de la elaboración de una propuesta de 
investigación científica, un 97 \% ( $\mathrm{f}=230$ ) reveló que sí. Se analizaron por género las respuestas para determinar si había diferencia significativa en las opiniones de lo que esperaban aprender entre mujeres y varones, y se observó que no existía ninguna diferencia ( $p>0.05$ ) atribuible al género; mismos resultados se dieron cuando se ejecutó el análisis por carrera que seguían los alumnos y alumnas ( $p>0.05)$.

Por otra parte, cuando se les interrogó sobre quiénes deberían tomar un curso de Metodología Científica, el 31,2 \% ( $\mathrm{f}=74$ ) de los participantes contestó que debería ser solo para los que pensaran dedicarse a la investigación, mientras que $68,8 \%$ ( $\mathrm{f}=163$ ) mencionó que no; es decir, que debe ser para todos los estudiantes indistintamente de sus preferencias académicas. Asimismo, al interrogárseles si es conveniente que todos los estudiantes de una Facultad de Ciencias tomen un curso de Metodología Científica, estos argumentaron, con un $85,2 \%$ ( $\mathrm{f}=202$, que sí deberían y el 14,8\% ( $\mathrm{f}=35$ ) adujo que no. En este sentido, se les cuestionó si todos los alumnos de licenciatura de una universidad deberían cursar la asignatura de Metodología Científica, a lo cual el $51,1 \%$ ( $\mathrm{f}=121)$ respondió que sí es conveniente y un 48,9\% ( $\mathrm{f}=116)$ dijo que no. Se examinaron por género las respuestas para establecer si acontecía diferencia significativa en estas de quienes deberían cursar la asignatura de Metodología Científica entre mujeres y varones, y se observó que no constaba diferencia ( $p>0.05)$ aplicable al género de los participantes; la misma situación sucedió cuando se realizó el examen por la carrera que cursaban los estudiantes ( $p>0.05$ ). Por último, se les cuestionó si durante su estancia como estudiantes universitarios se habían involucrado en algún proyecto de investigación antes de iniciar a cursar la asignatura de Metodología Científica, a los que respondió el 4,6 1\% ( $\mathrm{f}=11)$ que sí y el $95 \%,(\mathrm{f}=226)$, que no lo había realizado aún.

Expectativas de los estudiantes al final del curso

De los 237 estudiantes que inicialmente se encuestaron, hacia el final solo 177 decidieron completar la última evaluación, contando con un recaptura del $74,6 \%$ de los entrevistados, de los cuales el 40,1\% ( $\mathrm{f}=71$ ) correspondió a la carrera de QBP; seguido de las carreras de LBG, BIOL y LCA, con un 33,3\% ( $\mathrm{f}=59$ ), un $23,2 \%(\mathrm{f}=41)$ y un $3,4 \%(\mathrm{f}=6)$. Su edad varió entre una edad mínima de 17 a 31 años como máxima, con un promedio de 19,05 años y con una variación de 1,7 años de edad de los encuestados con respecto al valor medio encontrado. En relación con el género, el 44,6\% ( $\mathrm{f}=79)$ fue varones y el 55,3\% ( $\mathrm{f}=98)$, mujeres. Respecto a los cuestionamientos contemplados en la encuesta, cuando se les preguntó si habían aprendido historia de la ciencia, el 70,1 \% ( $\mathrm{f}=124)$ mencionó que no y el 29,9 1\% ( $\mathrm{f}=53)$ señalo que sí; comportamiento similar se presentó cunado se les interrogó si aprendieron filosofía de la ciencia, donde el 70,6\% ( $\mathrm{f}=125)$ dijo que no y el 29,4\% ( $\mathrm{f}=52)$, 
que sí. Cuando se les interpeló para que indicarán si aprendieron a elaborar un proyecto de investigación, un 94,6\% ( $\mathrm{f}=167$ ) mencionó que sí, y cuando se le interrogó si aprendieron a buscar información científica para elaborar un proyecto de investigación, el 81,9\% $(\mathrm{f}=145)$ expresó que sí. Al realizar el análisis por género de las contestaciones hechas para determinar si había diferencia significativa en las respuestas de lo que asimilaron entre mujeres y varones, se notó que no había ninguna diferencia ( $p$ > 0.05) imputable al género; los mismos resultados se dieron cuando se produjo el análisis por carrera que seguían los estudiantes ( $p>0.05)$.

En referencia al cuestionamiento relacionado sobre quiénes deberían cursar la asignatura de metodología científica, el 29.9\% ( $\mathrm{f}=53$ ) de los encuestados advirtió que solo aquellos que pretenden dedicarse a la investigación, mientras que el 70,1 1\% ( $\mathrm{f}=124)$ consideró que no. Además, al cuestionarles si todos los alumnos de una Facultad de Ciencia deberían tomar un curso de metodología científica, el 85,3\% ( $\mathrm{f}=151)$ mencionó que sí y el 14,7\% (f=26), que no. En este mismo orden de ideas, se les preguntó si es conveniente que todos los alumnos de la universidad la cursen, y el 59,9\% (f=106) dijo que sí, mientras que el 40,1 1\% (f=71), que no. Se indagó si el género determinaba una diferencia en las respuestas y el resultado fue que 80 no ( $p>0.05)$; igual situación se replicó por carrera, y también no significativo ( $p>0.05)$.

\section{Discusión}

De acuerdo con García Sánchez (2008), en la educación profesional, particularmente en el contexto del proceso de aprendizaje, han retomado suma importancia los componentes relacionados con las actitudes, motivaciones, conocimientos anteriores y expectativas que manifiestan los estudiantes universitarios. El mismo autor señala además que han servido estos estudios principalmente para mejorar los programas académicos, adecuar cambios curriculares y enriquecer el desempeño académico. Esencialmente porque "muchos programas docentes no siempre son consecuentes con las necesidades e intereses del alumnado a quien va dirigido" (Madrid, 2003, p. 146). En este contexto, la Universidad Autónoma de Nuevo León (2011) destaca el aprendizaje significativo que propone David Ausubel, el cual depende de la estructura cognitiva que el alumno posea, es decir, considera muy relevante lo que el alumno ya sabe,y establece así un vínculo con aquello que aprenderá, como lo propone Méndez (1993).

La presente investigación ha abordado los conceptos previos que los alumnos universitarios poseían, con lo cual se ha procurado durante el curso impartido enriquecer su estructura mental, examinando en el marco de una jerarquía conceptual la construcción de significados (Bruning et al., 2012), con propósitos cognoscitivos de enseñanza que han buscado un alineamiento cons- 
tructivo de comprensión, conocimiento, análisis y aplicación del conocimiento, de acuerdo con los objetivos cognitivos trazados en el programa de estudios (Ordoñez-Sierra y Rodríguez-Gallego, 2014). No obstante, los resultados de esta evaluación identifican fortalezas y debilidades en los componentes de este programa de Metodología Científica.

Se corroboró que el género y la carrera que cursaban los participantes no determinaron diferencias significativas en la opinión de estos, tanto al inicio como al final del estudio. De forma particular, en relación con los cuestionamientos, los estudiantes principalmente esperaban aprender a desarrollar un protocolo de investigación (99,6\%). Al respecto, Lacueva (1998) advierte que la enseñanza a través de la realización de proyectos es sumamente importante porque en este proceso se conjuga el estudio empírico con proposiciones o actividades en función de la realidad social, lo cual finalmente se llevó a cabo como parte de su ejercitación académica dentro de la unidad de metodología científica. Esta apreciación se corroboró al final del curso en el 94,6\% de los participantes, que aseveraron que sí lo realizaron. A esto se le aúna que también se reconoció el hecho de aprender a realizar búsquedas de información científica para localizar fuentes primarias o secundarias para elaborar los proyectos, como se había considerado previamente por los estudiantes.

En relación con el aprendizaje de historia de la ciencia, que es sumamente relevante como lo esperaban los participantes en el estudio $(71,3 \%$ ) y como también lo mencionan Scandroli y Eyler (2007), ya que este tópico ofrece una pauta de reflexión para comprender la estructuración de la ciencia e incluso contribuye a desarrollar un pensamiento crítico este no fue cumplido al final del curso, ya que solo el 29,9\% estuvo satisfecho. Lo anterior quizás radicó en el hecho de que este tema solo se imparte de manera introductoria y abreviada al comienzo de la unidad académica; el mismo comportamiento se suscitó en lo referente al aprendizaje de la temática de filosofía de la ciencia.

Por otra parte, en lo que atañe a quién debería cursar la asignatura de Metodología Científica, se observó como idea generalizada de los participantes que debe impartirse a todos los estudiantes de una Facultad de Ciencias, que es coincidente con lo que advierten BenZvi y Gai (1994) y Gutiérrez Vargas (2002), que resaltan la importancia en los estudiantes de educación superior de la formación del razonamiento científico y el desarrollo de las competencias para generar y utilizar la producción científica documentada. Escenarios similares se constituyeron cuando se les interrogó si esto es solo importante para los que deberían dedicarse a la investigación y si esto incumbiría el ser generalizado a todo el alumnado de una institución de educación superior.

En este contexto, de acuerdo con Acuña et al. (2009), en el marco de la enseñanza se hace constar de dos hechos 
relevantes y pertinentes por considerar. Primero cómo el profesor modela la identificación de los aspectos teóricos que serán impartidos; segundo, cómo retroalimenta al estudiante con hechos materiales que ilustren ese mismo conocimiento. El hallazgo que surge en este estudio, para que lo anteriormente se cumpla, es que el profesor tenga un perfil de investigador y además que sea competente en la planeación e instrumentación de interacciones didácticas que relacionen los aspectos teóricos con la realidad existente en sus ámbitos profesional y académico. Recordemos que "la formación de estudiantes competentes en el ámbito científico y tecnológico se enfrenta a la condición de la vigencia del conocimiento y su modificación continua" (Acuña et al., 2009, p. 101). La ciencia y sus métodos están en una constante estructuración y reestructuración del conocimiento, en procesos vinculados invariablemente con la realidad en espacio y tiempo; por lo tanto, es pertinente conocer las percepciones y valoraciones de los estudiantes que matizan sus intereses sobre una asignatura de aprendizaje,y, como señalan Paoloni et al. (2015, p. 49), con la finalidad de "[...] orientarlos en relación con lo que es posible esperar, lo que podemos brindarles en relación con sus expectativas y lo que podemos enseñarles -en el sentido más amplio del término- a valorar respecto de lo 82 deseable y lo posible" (p. 49).

Así pues, con la interacción que se sucede entre las expectativas de los estudiantes y la interpretación de los docentes, surge de manera particular la concreción de los aprendizajes. En este caso, se proyectó mejorar la alfabetización científica de los alumnos a partir de sus expectativas, es decir, ayudarlos a comprender los conceptos fundamentales de la ciencia, entender la naturaleza de la ciencia, como también la importancia de la ciencia y tecnología en la sociedad. Se trazó además con conocimientos prácticos el hecho que voluntariamente y no por cumplimiento académico acojan en sus vidas profesionales en formación el pensamiento científico, para que este influya más adelante en ampliar las opciones en su futuro actuar profesional. En este sentido, Hernández y Rodríguez (2015) advierten que en México "[...] los planes nacionales y programas sectoriales alientan la idea de que la educación superior debe encaminarse a ofrecer un capital humano formado para una inserción exitosa en las dinámicas laborales de la globalización y la sociedad del conocimiento" (p. 36).

Sobre la base del trabajo llevado a cabo y lo expuesto habrá que seguir impulsando una enseñanza sustentada en el interés y en los pensamientos de los estudiantes que brinde logros, tanto profesionales como cognoscitivos. Además, la constatación de las expectativas en los alumnos durante este estudio ha permitido avalar lo señalado por Gargallo López et al. (2011), al mencionar que esto se constituye en "[...] un claro reto para los profesores universitarios, que debemos potenciarlas en los estudiantes, por una 
parte, porque éstos aprenderán más y mejor, y por otra parte porque así es mucho más probable que les vaya bien en los estudios" (p. 216).

Sin duda, en el marco de las expectativas que los alumnos tuvieron hacia la asignatura de Metodología Científica prevalecieron intereses, experiencias previas e incluso la idiosincrasia personal. Finamente, fundados en la presente investigación, coincidimos con Steinmann et al. (2013), al afirmar que este tipo de investigaciones deben seguir avanzando, sobre lo que podríamos denominar la caracterización de la relación estudiante-docente, en la cual la voz de los estudiantes sea cada vez más imprescindible para la mejora de los planes curriculares de las asignaturas.

\section{Referencias}

Acuña, K.F., Irigoyen, J.J. y Jiménez, M. Y. (2009). Análisis de la percepción del desempeño docente en profesores de ciencias. En J. Varela, F. Cabrera y J. J. Irigoyen (Eds.), Estudios sobre comportamiento y aplicaciones. Volumen I (pp. 96-131). Guadalajara. Universidad de Guadalajara.

Ben-Zvi, N. y Gai, R. (1994). Macro-and micro- chemical comprehension of realworld phenomena. Journal of Chemical Education, 71(9), 730-732.

Bonomo Alciaturi, D.M., Lambert, J.I., Leal, G.A. y Espíndola, J.H. (2006). Cursos de metodología de la investigación y trabajos científicos en estudiantes de odontología. Revista de Posgrado de la VIa Cátedra de Medicina, (158), 1-4.
Bruning, R.H., Schraw, G.J. y Norby, M.M. (2012). Psicología cognitiva y de la instrucción. Madrid: Pearson Educación.

Campos Arenas, A. (2007). Pensamiento critico. Técnicas para su desarrollo. Bogotá. Magisterio.

Cantú-Martínez, P.C. (2012). Ciencia y conciencia humana. En P. C. CantúMartínez (Ed.), Sustentabilidad científica. Introversión sobre la ciencia, conciencia y racionalidad social (pp. 15-23). México, D. F: Clave Editorial, Universidad Autónoma de Nuevo León.

Díaz, V.P. (2011). Relación entre sociedad del conocimiento, metodología de la investigación científica y producción científica estudiantil en estudiantes de medicina. Colombia Médica, 42(3), 388-399.

García Sánchez, M.D. (2008). Expectativas de los estudiantes ante la asignatura de historia de la psicología. Enseñanza e Investigación en Psicología, 13(1), 15-26.

Gargallo López, B., Almerich Cerveró, G., García Félix, E. y Jiménez Rodríguez, M.A. (2011). Actitudes ante el aprendizaje en estudiantes universitarios excelentes y en estudiantes medios. Teoría de la Educación. Educación y Cultura en la Sociedad de la Información, 12(3), 200-220.

Gutiérrez Vargas, M.E. (2002). El aprendizaje de la ciencia y de la información científica en la educación superior. Anales de Documentación, (5), 197-212.

Hernández, J.M. y Rodríguez, J. (2015). La pertinencia de la educación desde la perspectiva de los estudiantes en una universidad pública mexicana. Cuadernos de Investigación Educativa, 6(1), 33-51. 
Lacueva, A. (1998) La enseñanza por proyectos: ¿mito o reto? Revista Iberoamericana de Educación, (16), 165-190.

Madrid, D. (2003). Intereses, necesidades y expectativas del alumnado de Magisterio de Lengua Extranjera (Inglés) durante su formación inicial. En M. Moreno y M. Escribano (Coords.), Tadea seu Liber de Amicitia (pp. 145-166). Granada: Grupo Editorial Universitario.

Méndez, Z. (1993). Aprendizaje y cognición. San José: Editorial Universidad Estatal a Distancia.

Molina Ordoñez, J., Huamani, Ch. y MaytaTristán, P. (2008). Apreciación estudiantil sobre la capacitación universitaria en investigación: Estudio preliminar. Revista Peruana de Medicina Experimental y Salud Pública, 25(3), 325-329.

Ordoñez-Sierra, R. y Rodríguez-Gallego, M.R. (2014). Expectativas del colectivo estudiantil de la Universidad de Sevilla. Profesorado, Revista de Currículum y Formación del Profesorado, 18(3), 393-410.

Paoloni, P.V., Martín, R.B. y Chiecher, A.C. (2015). Contextos de aprendizaje en el nivel medio y en la universidad. Percepciones y expectativas de estudiantes que ingresan en carreras de ingeniería. Formación Universitaria, 8(6), 47-56.
Petrucci, D. y Dibar Ure, M.C. (2001). Imagen de la ciencia en alumnos universitarios: Una revisión y resultados. Enseñanza de la Ciencia, 19(2), 217-229.

Tamez-Guerra, R.S. y Martínez-Rizo, F. (2012). Las reformas que necesita la educación mexicana. México, D. F: Edición No Venal.

Rué, J. (2009). El aprendizaje autónomo en educación superior. Madrid: Narcea.

Scandroli, N. y Eyler, N. (2007). Imagen de ciencia en alumnos de nivel universitario: Dimensión "método científico". Revista Iberoamericana de Educación, 44(3), 1-11.

Steinmann, A., Bosch, B. y Aiassa, D. (2013). Motivación y expectativas de los estudiantes por aprender ciencias en la universidad. Un estudio exploratorio. Revista Mexicana de Investigación Educativa, 18(57), 585-598.

Universidad Autónoma de Nuevo León (2005). Formación General universitaria de los estudiantes de licenciatura, profesional asociado y técnico superior universitario. México, D. F: autor.

Universidad Autónoma de Nuevo León (2011). Modelo Académico de Licenciatura. México, D. F: autor.

Woolfolk, A. (2010). Psicología educativa. México, D. F.: Pearson Educación. 